\title{
Altered protease and antiprotease balance during a COPD exacerbation contributes to mucus obstruction
}

Shashi Chillappagari, 2,3,7, Jenni Preuss ${ }^{3}$, Sebastian Licht ${ }^{3}$, Christian Müller ${ }^{3}$, Poornima Mahavadi ${ }^{2,7}$, Gaurav Sarode ${ }^{5,6}$, Claus Vogelmeier ${ }^{3}$, Andreas Guenther ${ }^{2,7,8,9}$, Lutz Nahrlich ${ }^{1,7}$, Bruce K. Rubin ${ }^{4}$ and Markus O. Henke $e^{3,5,6^{*}}$

\begin{abstract}
Background: Proteases have been shown to degrade airway mucin proteins and to damage the epithelium impairing mucociliary clearance. There are increased proteases in the COPD airway but changes in protease-antiprotease balance and mucin degradation have not been investigated during the course of a COPD exacerbation. We hypothesized that increased protease levels would lead to mucin degradation in acute COPD exacerbations.

Methods: We measured neutrophil elastase (NE) and alpha 1 protease inhibitor (A1-PI) levels using immunoblotting, and conducted protease inhibitor studies, zymograms, elastin substrate assays and cigarette smoke condensate experiments to evaluate the stability of the gel-forming mucins, MUC5AC and MUC5B, before and 5-6 weeks after an acute pulmonary exacerbation of COPD ( $n=9$ subjects).

Results: Unexpectedly, mucin concentration and mucin stability were highest at the start of the exacerbation and restored to baseline after 6 weeks. Consistent with these data, immunoblots and zymograms confirmed decreased NE concentration and activity and increased A1-PI at the start of the exacerbation. After recovery there was an increase in NE activity and a decrease in A1-PI levels. In vitro, protease inhibitor studies demonstrated that serine proteases played a key role in mucin degradation. Mucin stability was further enhanced upon treating with cigarette smoke condensate (CSC).
\end{abstract}

Conclusion: There appears to be rapid consumption of secreted proteases due to an increase in antiproteases, at the start of a COPD exacerbation. This leads to increased mucin gel stability which may be important in trapping and clearing infectious and inflammatory mediators, but this may also contribute acutely to mucus retention.

Keywords: COPD, Mucin, Proteases, Alpha-1-protease inhibitor, Neutrophil elastase, Cigarette smoke, Hypersecretion

\section{Introduction}

Proteases play a major role in bacterial entrapment [5], pathogen phagocytosis [16], mucin hypersecretion and mucociliary clearance [9]. In COPD there is a deficiency and decreased activity of anti-proteases [21, 30], contributing to emphysema [1] and mucus hypersecretion [4]. This protease and anti-protease imbalance has been suggested to result from neutrophil infiltration in the lung $[3,28]$.

\footnotetext{
* Correspondence: m.henke@asklepios.com

${ }^{3}$ Department of Pulmonary Medicine, Philipps-University Marburg,

Baldingerstrasse 1, Marburg 35043, Germany

${ }^{5}$ Asklepios Fachkliniken München-Gauting, Robert-Koch-Allee 2, Gauting 82131, Germany

Full list of author information is available at the end of the article
}

These neutrophils release proteases including neutrophil elastase (NE), cathepsin-G (CG), and proteinase 3 (PR3).

Mucins are linearly linked core proteins encoded by mucin (MUC) genes. The principal airway gel-forming mucins are MUC5AC and MUC5B [24]. Several lines of evidence show that mucus is hypersecreted in COPD [30]. Studies performed on surgically isolated lung tissues from COPD patients have shown that mucus containing inflammatory exudate accumulates in small airways and is associated with disease progression [11]. In biopsies from COPD patients with severe lung disease, mucus occupies about $15 \%$ of the total luminal area of small airways; whereas, in "healthy" smokers, it is limited to less than 
$5 \%$ of the luminal area [13]. The amount of small airway mucus is strongly associated with mortality in patients with COPD [12]. We have shown that increased secretion of serine proteases in cystic fibrosis (CF) can degrade the gel-forming mucins during the time of transport from peripheral airways to central airways [9]. However the effect of serine proteases on mucin in COPD subjects has not been well characterized.

In this study, we investigated the role of proteases and anti-proteases on COPD mucin stability and degradation during the course of an infectious and inflammatory exacerbation of COPD. As in subjects with CF, we hypothesized that that there would be an acute increase in proteases during exacerbation leading to mucin degradation.

\section{Methods}

\section{Subject details and sample collection}

Nine subjects were included in the study, with a mean age of 59.9 years. They had been hospitalized or evaluated in the outpatient clinic of the Department of Pulmonary Medicine, Philipps-University Marburg, because of an acute pulmonary exacerbation of COPD defined by the Anthonisen criteria of increased dyspnea and cough, increased sputum volume and change in sputum color [2]. Subjects were included if they had at least 2 of symptoms with an onset within 7 days before the start of the study. We included only subjects in GOLD group II or III (FEV1/VC $<70 \%$, FEV1 30-80 \%) [31]. Criteria for exclusion were signs of bacterial infection with fever $>38.5{ }^{\circ} \mathrm{C}$, CRP-elevation > $30 \mathrm{mg} / \mathrm{L}$ or procalcitonin elevation $>5 \mu \mathrm{g} / \mathrm{L}$, suspected or known pneumonia with infiltrate on chest $\mathrm{x}$-ray, Pseudomonas aeruginosa in sputum cultures, pre-admission antibiotic treatment, or suspected or known asthma. At the first day of the reported pulmonary exacerbation symptoms, sputum was collected. All subjects were followed up 5-6 weeks after the onset of the exacerbation and another sputum sample was collected (Table 1). At visit 1 the subjects were grouped as "COPD with exacerbation" and after 5-6 weeks (visit 2) as "COPD without exacerbation". All subjects were treated with oral steroids (40 mg once daily) for total of 10 days, and inhalation therapy with long-acting muscarinic antagonists and short- and long-acting beta2-agonists. Five of the 9 subjects were current smokers and 4 were former smokers. Antibiotic treatment was not necessary for any of the subjects and all

Table 1 Study summary

\begin{tabular}{lll}
\hline & Time & Procedures \\
\hline Visit 1 & $\begin{array}{l}\text { day 1; week 1 (within 7 days } \\
\text { after start of exacerbation) }\end{array}$ & $\begin{array}{l}\text { Sputum collection, pulmonary } \\
\text { function test, chest x-ray, blood } \\
\text { sample, physical examination }\end{array}$ \\
Visit 2 days 40-46, week 5-6 & $\begin{array}{l}\text { Sputum collection, pulmonary } \\
\text { function test, blood sample }\end{array}$ \\
\hline
\end{tabular}

of them recovered from the exacerbation within the observed time. Clinical characteristics and demographics of the COPD subjects are given in Table 2. Sputum collection was approved by the Philipps-University Marburg Institutional Review Board.

\section{Control mucus collection}

As a control group we collected mucus coating the endotracheal tubes (ETT) of subjects who had no lung disease and required non-thoracic surgery under general anesthesia. When the subject was extubated, the ETT was removed from the airway and mucus was removed by gently scraping the ETT $[25,26]$. Collected ETT mucus was placed in a small O-ring container to prevent dehydration, labeled as to date of collection with no subject identifiers, and sent to Philipps-University Marburg on dry ice. ETT mucus collection was approved by the Virginia Commonwealth University Institutional Review Board and signed consent, and assent when appropriate, was obtained.

\section{Protease inhibitors and antibodies}

$\mathrm{NE}$ and cathepsin $\mathrm{G}$ were purchased from Merck Chemical, Nottingham, UK. Serine protease inhibitors diisopropyl fluorophosphates (DFP), phenylmethyl sulfonyl fluoride (PMSF), and 1-chloro-3-tosylamido-7-amino-2-heptanone $\mathrm{HCl}$ (TLCK), metalloprotease (EDTA and GM6001) and cysteine proteases (leupeptin and E64) were purchased from Sigma (Saint Louis, MO). Alpha-1 protease inhibitor (A1-PI) was obtained as Prolastin ${ }^{\bullet}$ (Grifols Therapeutics Inc. Frankfurt, Germany) and was used at a final concentration of $0.3 \mu \mathrm{g} / \mathrm{mL}$. DFP (final concentration $2 \mathrm{mM}$ ); PMSF (final concentration $2 \mathrm{mM}$ ); TLCK (final concentration $10 \mathrm{mM}$ ); EDTA (final concentration $100 \mathrm{mM}$ ); E64 (final concentration $500 \mathrm{ng} / \mathrm{mL}$ ) or Merck Chemical (Nottingham, UK): GM6001 (final concentration $40 \mu \mathrm{M}$ ) and leupeptin (final concentration $40 \mu \mathrm{M}$ ) were used. Polyclonal anti-MUC5AC and anti-MUC5B antibodies were generated as previously described [10]. The antibodies were characterized and specificity was ascertained by pre-absorption studies using increasing concentrations of the antigenic peptides [25]. Specificity of these antibodies was verified using immunoblotting against MUC5AC and MUC5B from whole cell lysates, secretions from normal human tracheobronchial epithelial (NHBE) cells (passage 2) (Clonetics Corp., La Jolla, CA, USA), and human mucus. The blots were analyzed with antisera for MUC5AC and MUC5B and the pre-immune sera of the same rabbit. We found one well-defined band of high molecular weight with the antisera. To increase the specificity of the antibodies and reduce nonspecific binding, affinity purification of the antipeptide antibody was performed from the whole serum using the immobilized amino acid sequences of interest (SulfoLink-Kit, Pierce). An internal control for mucin was 
Table 2 Demographic data of the COPD patients included the study

\begin{tabular}{|c|c|c|c|c|c|c|c|c|c|c|c|c|}
\hline subject & age & $\begin{array}{l}\text { Pack } \\
\text { years }\end{array}$ & smoking status & & $\begin{array}{l}\text { chest x-ray } \\
\text { (infiltrations?) }\end{array}$ & $\begin{array}{l}\text { CRP in } \\
\mathrm{mg} / \mathrm{l}\end{array}$ & $\begin{array}{l}\text { leucocytes in G/l } \\
\text { (normal: 4.3-10) }\end{array}$ & $\begin{array}{l}\text { Procalcitonin } \\
\text { in } \mu \mathrm{g} / \mathrm{l}\end{array}$ & $\begin{array}{l}\text { FEV1 } \\
(\%)\end{array}$ & $\begin{array}{l}\text { VC } \\
(\%) \\
\end{array}$ & $\begin{array}{l}\text { FEV1/ } \\
\text { VC (\%) }\end{array}$ & color of sputum \\
\hline \multirow[t]{2}{*}{01} & 51 & 80 & current smoker & Visit 1 & no & $<5$ & 6.24 & $*$ & 49 & 95 & 47 & clear \\
\hline & & & & Visit 2 & & $<5$ & 7.05 & * & 58 & 94 & 55 & clear \\
\hline \multirow[t]{2}{*}{02} & 61 & 80 & former smoker & Visit 1 & no & $<5$ & 8.28 & 0.22 & 63 & 95 & 66 & clear/slightly yellow \\
\hline & & & & Visit 2 & & 14 & 8.14 & 0.17 & 67 & 95 & 70 & clear \\
\hline \multirow[t]{2}{*}{03} & 74 & 20 & former smoker & Visit 1 & unkown & 31 & 7.25 & * & 67 & 95 & 70 & clear \\
\hline & & & & Visit 2 & & 18 & 6.64 & $*$ & 81 & 95 & 75 & clear \\
\hline \multirow[t]{2}{*}{04} & 67 & 20 & former smoker & Visit 1 & no & 11 & 10.7 & $<0.1$ & 48 & 89 & 53 & clear/slightly yellow \\
\hline & & & & Visit 2 & & * & * & * & 42 & 98 & 43 & clear \\
\hline \multirow[t]{2}{*}{05} & 65 & 30 & former smoker & Visit 1 & no & 18 & 3.92 & $<0.1$ & 68 & 98 & 69 & clear \\
\hline & & & & Visit 2 & & $<5$ & 5.21 & $<0.1$ & 95 & 98 & 97 & clear \\
\hline \multirow[t]{2}{*}{06} & 52 & 50 & current smoker & Visit 1 & no & 11 & 13 & $*$ & 32 & 93 & 34 & clear \\
\hline & & & & Visit 2 & & $<5$ & 9.15 & * & 74 & 98 & 75 & clear \\
\hline \multirow[t]{2}{*}{07} & 57 & 35 & current smoker & Visit 1 & no & $<5$ & 5.89 & $*$ & 37 & 91 & 41 & clear \\
\hline & & & & Visit 2 & & * & $*$ & * & 58 & 90 & 64 & clear \\
\hline \multirow[t]{2}{*}{08} & 50 & 50 & current smoker & Visit 1 & no & 14 & 15.3 & $<0.1$ & 36 & 93 & 39 & clear \\
\hline & & & & Visit 2 & & 24 & 15.3 & $<0.1$ & 51 & 92 & 55 & clear \\
\hline \multirow[t]{2}{*}{09} & 62 & 70 & current smoker & Visit 1 & no & 7 & 8.5 & * & 56 & 89 & 63 & clear/slightly yellow \\
\hline & & & & Visit 2 & & $<5$ & 6.15 & * & 67 & 89 & 75 & clear \\
\hline
\end{tabular}

*Data not collected

collected from a voluminous sputum sample from a single patient undergoing lung transplantation for non-CF bronchiectasis [10]. Mucin signals obtained from COPD sputum and normal controls were normalized to this internal control, which was set to $100 \%$.

\section{Agarose wet western blotting for MUC5AC and MUC5B}

Sputum and internal control samples were diluted 1:10 with PBS and denatured using Laemmli buffer (125 mM Tris pH 6.8; 4 \% SDS; 20 \% glycerol; $0.001 \%$ bromophenol blue, $20 \mathrm{mM} \mathrm{DTT}$ ) and separated using $1 \%$ agarose gels $(15 \times 15 \mathrm{~cm})$, prepared in running buffer $(25 \mathrm{mM}$ Tris, $250 \mathrm{mM}$ glycine, $0.1 \%$ SDS). Electrophoresis was performed in a horizontal gel apparatus at $60 \mathrm{~V}$ at room temperature for the first $30 \mathrm{~min}$, and then voltage was set to $100 \mathrm{~V}$ for the rest of the time. To identify small proteins that remained in the gel, the gel was stopped when the dye front was $2 / 3$ of the distance from the wells. Proteins were transferred to nitrocellulose membranes using vertical wet electroblotting apparatus, LKB bromma at $(300 \mathrm{~mA})$ for $3 \mathrm{~h}$ at $4{ }^{\circ} \mathrm{C}$. Membranes were blocked with $10 \%$ nonfat skimmed milk in PBS for $1 \mathrm{~h}$ and subsequently incubated with primary antibodies (1:100 MUC5AC and 1:100 MUC5B) for $18 \mathrm{~h}$ in $1 \%$ nonfat skimmed milk in PBS at $4{ }^{\circ} \mathrm{C}$. Blots were washed 3 times in PBS for $10 \mathrm{~min}$, and incubated with the secondary HRP-labeled goat-anti-rabbit antibody (1:1000) (Jackson-Immuno) in 1 \% nonfat skimmed milk in PBS for $1 \mathrm{~h}$. Blots were washed in PBS for 10 min 3 times and developed using the Pico-Developer-Kit (Pierce). Exposures were taken on CL-XPosure film (Pierce) at equal exposure times. The films were scanned and band intensities were determined by densitometry using $\mathrm{NIH}$ Image software (http://rsbweb.nih.gov/nih-image/).

\section{SDS PAGE western blotting for alpha-1-protease inhibitor (A1-PI) NE}

Sputum and internal control samples were diluted (1:100 for A1-PI and 1:20 for NE) with PBS and homogenized using a syringe. As a positive control, human A1-PI $\left(\right.$ Prolastin $^{\circ}$ ) and a control subject sample known to contain $\mathrm{NE}$ were used. Upon denaturation, the samples were separated by SDS-PAGE (7.5 \% for A1-PI and $10 \%$ for HNE) before blotting onto PVDF membranes. The membranes were blocked with $5 \%$ nonfat skimmed milk in TBST for $1 \mathrm{~h}$ at room temperature and incubated with primary antibodies anti- A1-PI (Sigma Aldrich) HNE- (Abcam) over night at $4{ }^{\circ} \mathrm{C}$. Membranes were washed three times in TBST for $15 \mathrm{~min}$, and incubated with the HRP-labeled secondary antibodies dissolved in TBST containing $5 \%$ milk, for $1 \mathrm{~h}$ at room temperature. Membranes were developed using ImmunoCruz Luminol agent (Santa Cruz). Exposures were taken on CL-XPosure film (Pierce) at equal exposure times. The film was scanned and band densities were determined by densitometry using NIH Image software (http:// rsbweb.nih.gov/nih-image/). 


\section{Analyzing NE activity with specific substrates}

To analyze free NE activity in the sputum we used the substrate N-Methoxysucuccinyl -ALA-ALA-PRO -VAL P-Nitroanilide (Sigma Aldrich, Saint Louis, MO). According to the manufacturer, the substrate is specifically hydrolyzed by HNE and cannot be hydrolyzed by cathepsin G. To get comparable results, we used a test volume set at $1000 \mu \mathrm{L}$, consisting of $900 \mu \mathrm{L}$ substrate solution and $5 \mu \mathrm{L}$ of patient sputum samples with added buffer, adding the enzymatically active compound at last. The test solution was thoroughly mixed and the analysis was started immediately. As an internal control we used triplets of each sample in dilution steps of 1:10, 1:20 and 1:40 in PBS. As an external control $3 \mu \mathrm{L}$ of purified NE (Calbiochem ${ }^{\circ}$, product no. 324681) was used. The degradation of the substrate was analyzed at a wavelength of $410 \mathrm{~nm}$ over $30 \mathrm{~min}$ in a Nicolet Evolution $100 \mathrm{UV}$-vis Spectrophotometer. The results were documented via VISIONlife ${ }^{\text {twx }}$ software.

Analysis of protease activity using zymograms NOVEX 4-16 \% zymogram blue casein gels (Life Technologies) were used to detect NE enzymatic activity in sputum samples. Mucin samples were homogenized using a sterile Filtropur syringe filter $(0.20 \mu \mathrm{m}$ pore size). Equal volumes of homogenized sputum samples were loaded on a gel and separated using electrophoresis at $125 \mathrm{~V}$ for $2 \mathrm{~h}$. The gel was run in Tris/glycine SDS running buffer under nondenaturing conditions. The separated proteins were renatured using a buffer containing a non-ionic detergent (Novex Zymogram Renaturing Buffer). Gels were washed twice for $15 \mathrm{~min}$ in PBS and equilibrated using a developing buffer (Novex ${ }^{\circ}$ Zymogram Developing Buffer) containing divalent metal cations for $30 \mathrm{~min}$ as described in the manufacturer's protocol. The gel was then incubated at $37{ }^{\circ} \mathrm{C}$ for $20 \mathrm{~h}$ in fresh developing buffer for enhanced digestion. Enzymatic activity was visualized as a clear band against a dark background of stained casein. ETT mucus from healthy subjects was used as a positive control. The gels were scanned using a Canon ScanLide 50 scanner and activity was measured by quantification of digested area using Image-J densitometry software.

\section{Preparation of cigarette smoke condensate (CSC)}

Cigarettes were smoked in a smoking chamber for $5 \mathrm{~min}$ and smoke was suctioned using a vacuum pump into a
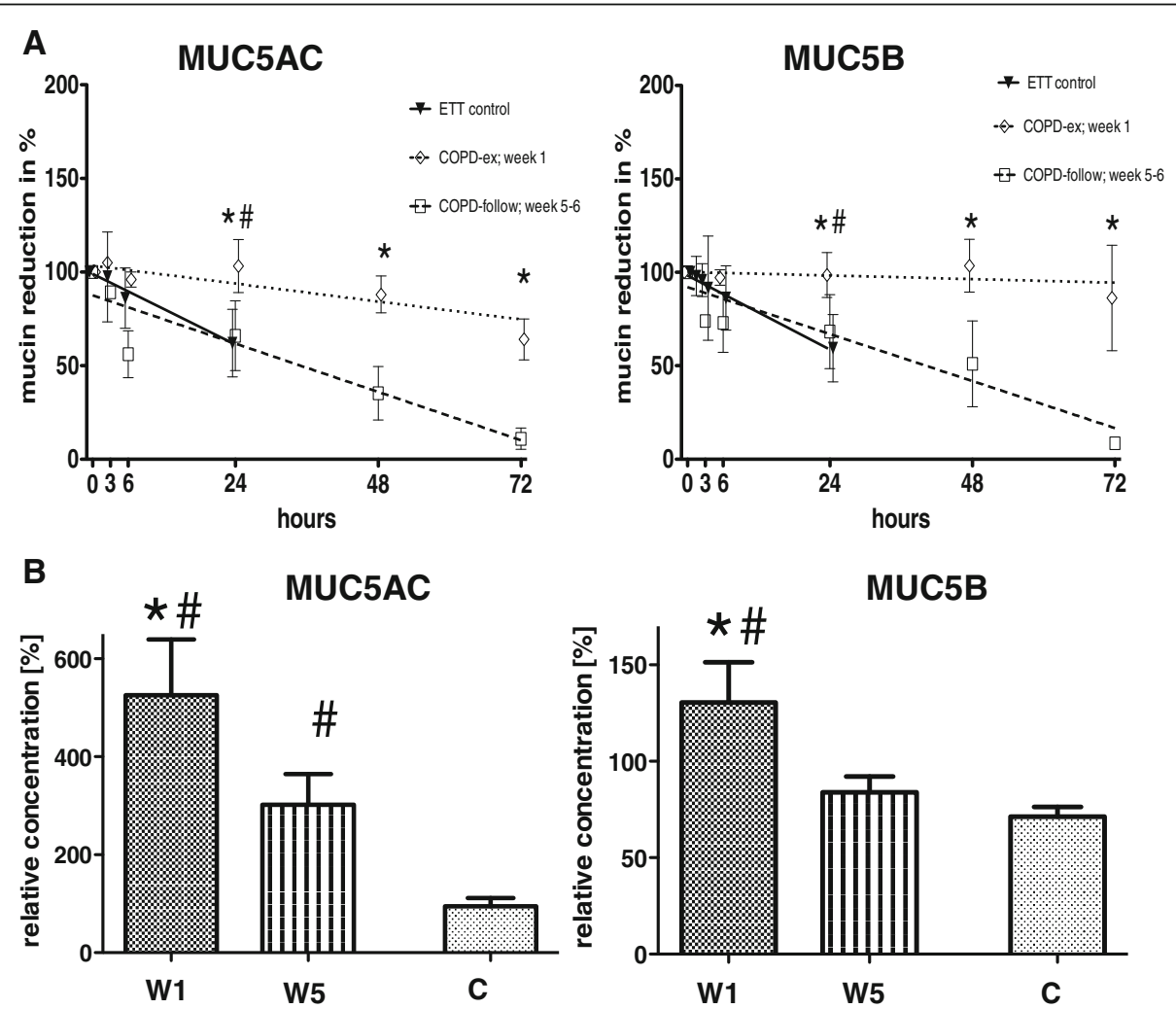

Fig. 1 Sputum collection and mucin quantification from COPD subjects. Quantification of mucin in sputum obtained from 9 subjects with COPD. a Sputum was collected at the start of an exacerbation of COPD (COPD-ex; week 1) and again 5-6 weeks later (COPD-follow; week 5-6) from the same subjects. Results were compared to mucin concentration in mucus from 11 ETT control mucus samples (control). $\mathbf{b}$ The results are shown as mean density of individual samples related to the internal control (=100 \% relative concentration). * significant to "COPD-follow; week 5-6" ( $t$-Test, $p<0.05)$; \# = significant to "control" (Mann Whitney test, $p<0.05)$ 
Falcon tube containing $37{ }^{\circ} \mathrm{C}$ pre-warmed $10 \mathrm{~mL}$ PBS. Care was taken to maintain constant temperature $\left(37^{\circ} \mathrm{C}\right)$ and continuous stirring to allow the smoke to dissolve fully in PBS. One cigarette in $10 \mathrm{~mL}$ PBS is referred to as CSC10, which was considered to be $10 \%$.

\section{Inhibition of NE-activity by CSC}

Inhibition studies of NE were performed spectrophotometrically using specific NE-substrate (Merck Chemicals) as described in the manufacturer protocols (Elastin). Different concentrations of CSC (CSC5, CSC10) were used to inhibit pure NE (final concentration $0.33 \mu \mathrm{g} / \mathrm{ml}$ ). Activity of $\mathrm{NE}$ was measured at $410 \mathrm{~nm}$.

\section{Data analysis}

All analyses were performed at least in triplicate. Results are presented as mean values \pm standard error. The mucin concentration of the sputum samples was normally distributed within all groups (Skewness $< \pm 2$ ). The mucin concentration is shown as \% to an internal control. To compare sputum samples from the same group (COPD week 1 and week 5-6) we used the paired $t$-Test. To compare sputum samples of the different groups we used the Mann-Whitney $U$-Test. After post hoc correction for multiple comparisons, a probability of $p<0.05$ was considered significant. All analyses were performed by means of GraphPad Prism 5 software (San Diego, CA). Descriptive statistics were used to summarize subject demographics.

\section{Results}

Increase in mucins concentration before exacerbation

Sputa were collected at the onset of a COPD exacerbation and 5-6 weeks later and compared with ETT mucus obtained from healthy subjects. Mucin stability in samples was analyzed in vitro after incubation at $37^{\circ} \mathrm{C}$ for $24 \mathrm{~h}$. In comparison to ETT mucus, there was a 5 -fold increase in MUC5AC and 2-fold increase in MUC5B at the start of an exacerbation. Five to 6 weeks later, MUC5AC was about 3-fold higher in comparison to ETT control mucus or 2-fold lower than at the start of the exacerbation. MUC5B concentration decreased to ETT mucin levels at 5-6 weeks (Fig 1a, b). Results obtained from immunoblot densitometry showed about a $40 \%$ degradation of sputa from COPD patients at 5-6 weeks with almost no degradation seen at the start of an exacerbation. These observations suggest that there was dramatically (and unexpectedly) increased antiprotease activity or decreased NE activity at the start of the exacerbation, but by week 5 , protease and antiprotease activity returns to baseline.
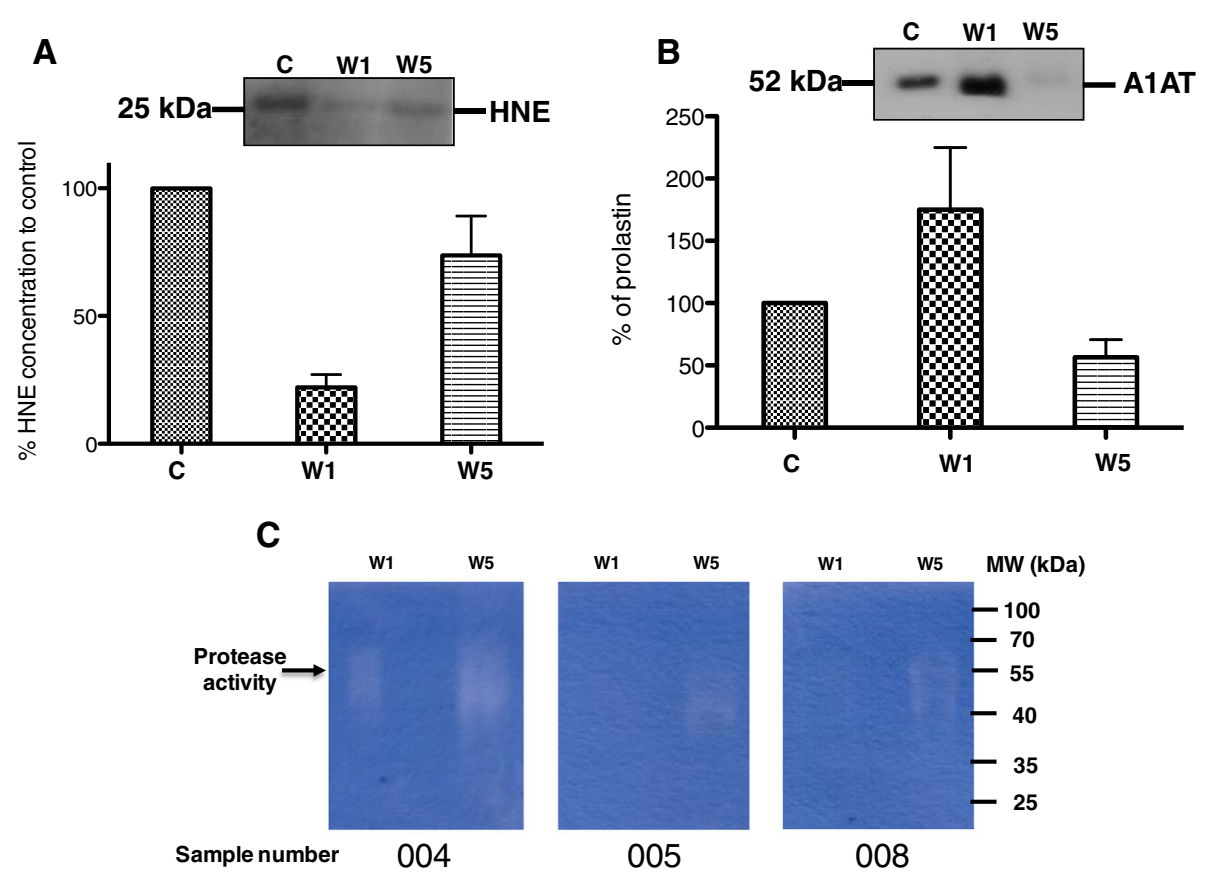

Fig. 2 Altered NE and A1-PI in COPD sputum samples. a Representative western blot analysis for NE from 3 COPD subjects $(003,005,007)$ during the course of exacerbation. $\mathrm{C}=$ Control, $\mathrm{W} 1=$ week $1, \mathrm{~W} 5=$ week $5-6$. To compare the results graphically western blots were analyzed by densitometry and compared to an internal control sputum sample, which was set to $100 \%$. b Representative western blot for A1-PI from 3 COPD subjects (003, 005, 007) $C=$ control; $W 1=$ week 1 and $W 5=$ week $5-6$. To compare the results graphically western blots were analyzed by densitometry and compared to an internal control sputum sample, which was set to 100. c Analysis of sputum protease activity by zymogram. Sputum was obtained from 3 COPD subjects with an acute exacerbation within the first week (COPD-ex; week 1) and from the same subjects 5-6 weeks later (COPD-follow; week 5-6) $n=3$ 


\section{A1-PI and free NE concentration in sputum}

In order to understand the reasons for increased mucin stability at the start of an exacerbation, we quantified $\mathrm{NE}$ and A1-PI in sputum samples using an immunoblot and found that the NE concentration in the sputum of the COPD patients at the onset was 3.5 times lower than at 5-6 weeks after the onset of the exacerbation (Fig 2a). Additionally, we analyzed nonspecific protease activity in sputa from 3 subjects with COPD at the start of an exacerbation and compared it to sputa from the same subjects 5-6 weeks later using zymograms (Fig. 2c). Dornase alfa was added to the sputum to release proteases trapped in DNA. The strongest signal for nonspecific enzyme activity was detected only in the sputum samples obtained 5-6 weeks after the onset. We found that A1-PI concentration in the sputum of the COPD patients at the beginning of the exacerbation was 3 times higher when compared with sputum 5-6 weeks after the onset of the exacerbation (Fig. 2b). Thus at the start of a
COPD exacerbation it appears that protease activity is low which as well correlates to increased A1-PI.

\section{Increase in A1-PI at the start of an exacerbation inhibits} mucin degradation

To verify the role of non-specific proteases in COPD mucin degradation 5-6 weeks after the onset of an exacerbation, we incubated the mucus with different protease inhibitors at $37{ }^{\circ} \mathrm{C}$ for $24 \mathrm{~h}$. Results obtained from immunoblots revealed that without protease inhibitors, MUC5AC concentration decreased by $96 \%$ and MUC5B by $95 \%$ of the initial concentration, whereas incubation with the protease inhibitors DFP, PMSF and TLCK inhibited mucin degradation. However, incubation with the metalloprotease inhibitors, EDTA and GM6001, and cysteine protease inhibitors, Leupeptin and E64, did not inhibit the mucin degradation (Fig. 3a). We also tested if A1-PI could decrease mucin degradation. We incubated COPD sputa from 4 subjects at week 5-6
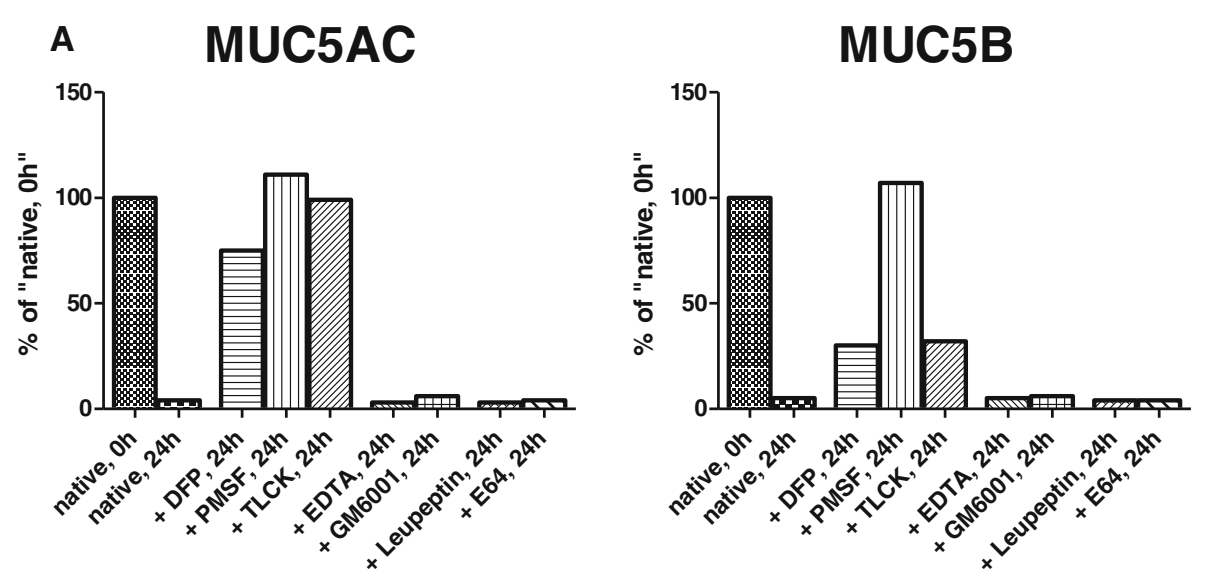

B $\quad$ MUC5AC
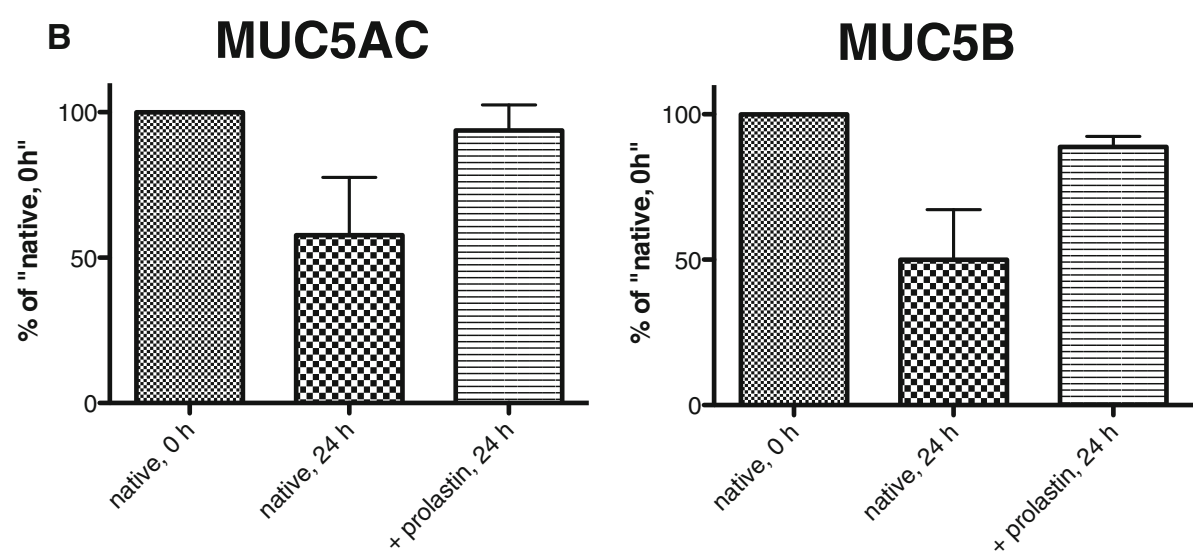

Fig. 3 Serine proteases inhibit mucin degradation. Analysis of sputum MUC5AC and MUC5B by western blot after incubation at $37^{\circ} \mathrm{C}$ over $24 \mathrm{~h}$ with or without of protease inhibitors. Sputum was obtained from a COPD subject 5-6 weeks after an acute exacerbation. Mucin concentration of the native control without incubation over $24 \mathrm{~h}$ was set to $100 \%$ a We used the serine protease inhibitors DFP, PMSF and TLCK, the metalloprotease inhibitors EDTA and GM6001 and the cysteine protease inhibitors leupeptin and E64. Analysis was performed in triplicate. b Incubation of COPD sputa (5-6 weeks after the onset) with A1-PI $(n=4)$ and compared with control sputa 
after exacerbation, with and without A1-PI and inhibited the degradation of MUC5AC to just $6 \%(\mathrm{SEM} \pm 9)$ and MUC5B to $11 \%(\mathrm{SEM} \pm 3)$ of the native mucin concentration (Fig. 3b).

\section{Inaccessibility of NE decreases mucin degradation}

Serial dilutions of mucus identified that a 1:60 to $1: 100$ dilution of mucus is most effective for measuring protease activity (Fig. 4a). We then incubated COPD sputum from the start of an exacerbation at dilutions of 1:60 and 1:80 with synthetic proteases, NE $0.02 \mathrm{mg} / \mathrm{mL}$, and cathepsin $\mathrm{G} 100 \mu \mathrm{U} / \mu \mathrm{L}$ and incubated at $37{ }^{\circ} \mathrm{C}$ for $6 \mathrm{~h}$. Both MUC5AC and MUC5B mucins were degraded by $\mathrm{NE}$ and cathepsin $\mathrm{G}$ in a concentration dependent manner (Fig. 4b).
Cigarette smoke condensate (CSC) decreases mucin degradation and inhibits protease activity

To analyze the role of CSC on mucin degradation, COPD sputum was incubated with different CSC concentrations (5-40\%) for 0, 24 and $36 \mathrm{~h}$. COPD sputa without CSC, MUC5AC and MUC5B mucins were significantly degraded after 24 and $36 \mathrm{~h}$. A dose dependent inhibition of mucin degradation was observed with COPD sputum incubated with increasing concentrations of CSC (Fig. 5a). To elucidate the role of CSC in inhibiting mucin degradation, we incubated $\operatorname{HNE}(0.33 \mu \mathrm{g} / \mathrm{mL})$ with different concentrations (5-10\%) of CSC and analyzed the activity of NE using a HNE specific substrate (5-methoxy-Ala-Ala-Pro-Val). To conclude, CSC directly interfered with protease activity in a dose dependent manner (Fig. 5b).
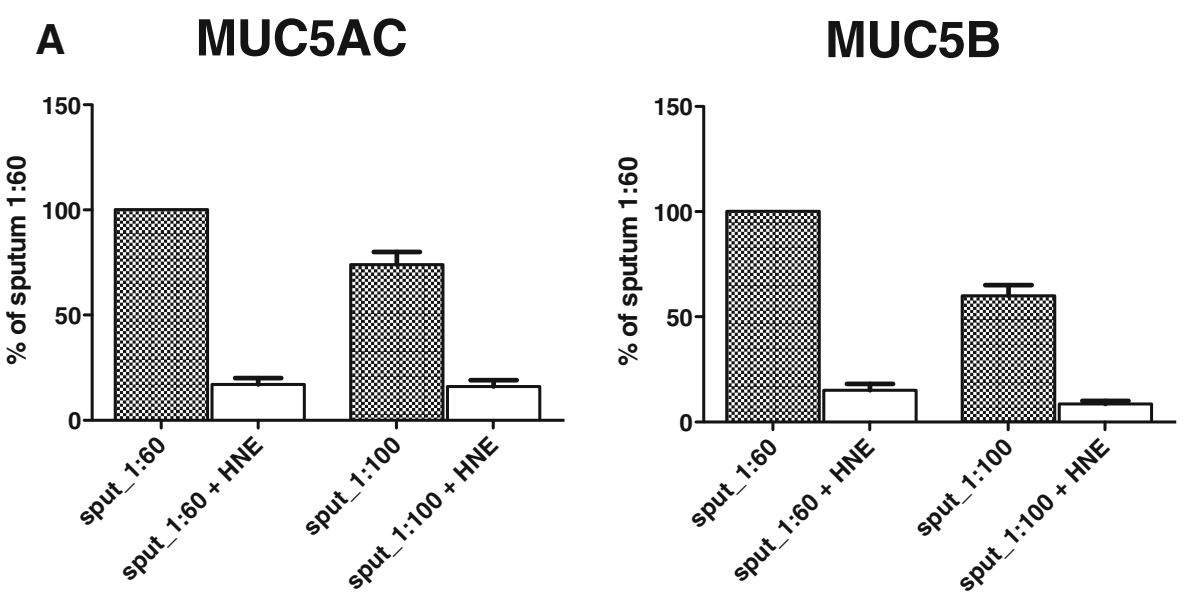

B MUC5AC

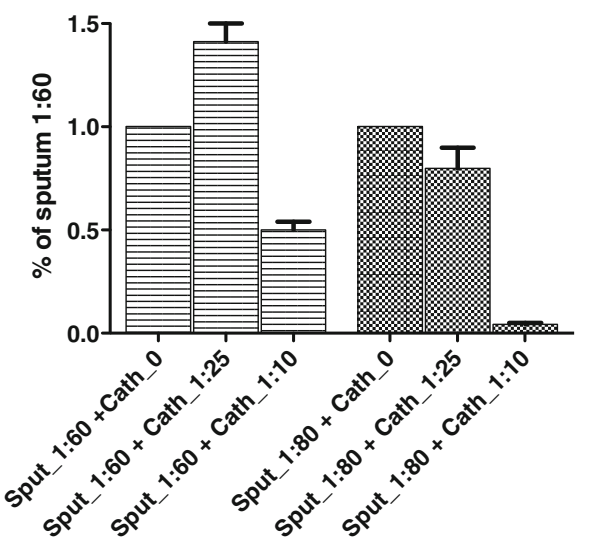

MUC5B

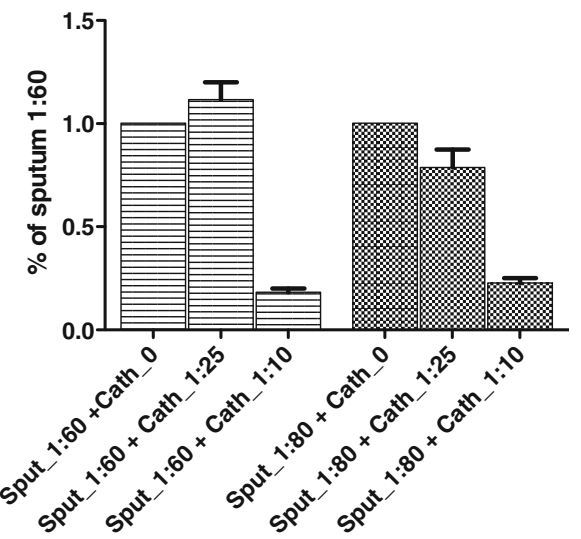

Fig. 4 Mucus hydration increases mucin degradation. a Sputum was obtained at the onset of a COPD exacerbation and diluted 1:60 and 1:100. Mucin degradation was measured after incubation for $6 \mathrm{~h}$ at $37^{\circ} \mathrm{C}$ with or without NE $0.02 \mathrm{mg} / \mathrm{mL}$. Mucin concentration of the control at 1:60 was set to $100 \%$. b Sputum was obtained at the onset of a COPD exacerbation and diluted 1:60 and 1:80. Mucin degradation was measured after incubation for $6 \mathrm{~h}$ at $37^{\circ} \mathrm{C}$ with cathepsin G $100 \mu \mathrm{U} / \mu \mathrm{L}$ diluted 1:25 and 1:10. Mucin concentration of the control was set at 1:60 and 1:80 was set to $100 \%$ 


\section{A MUC5AC}

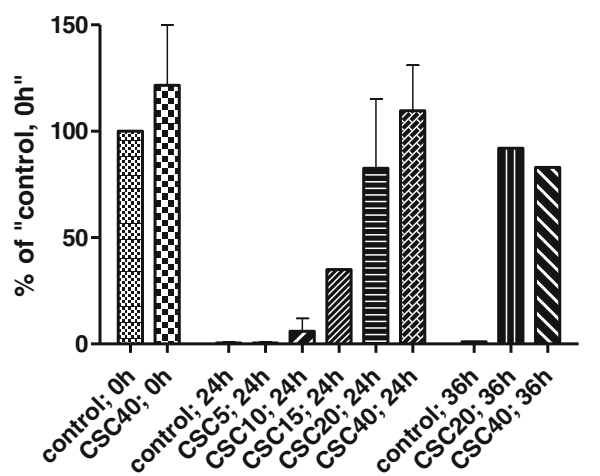

MUC5B

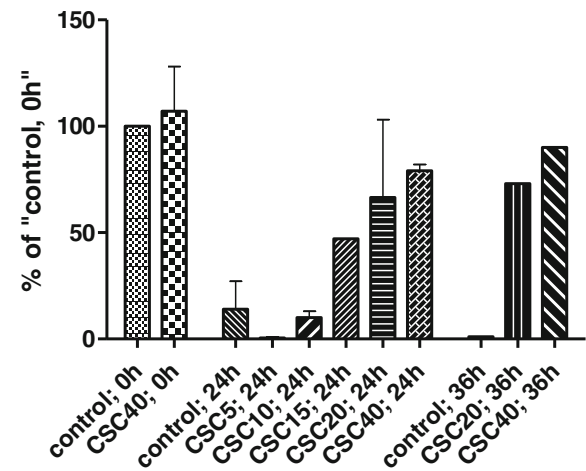

\section{B}

HNE-CSC-inhibition

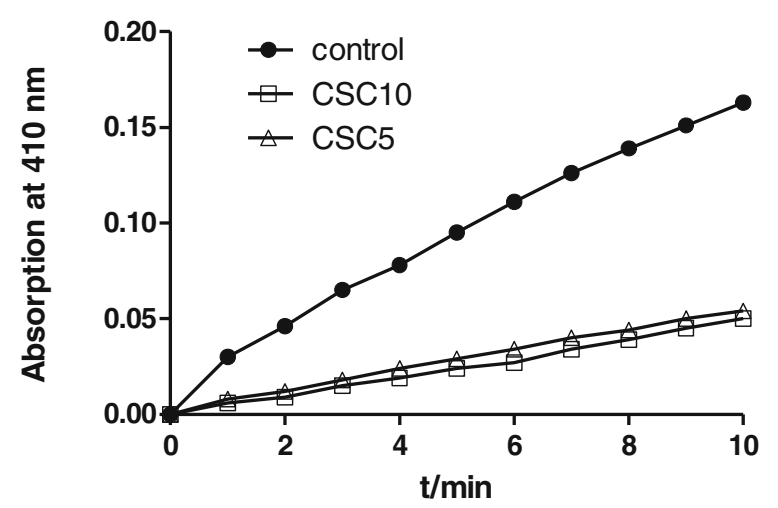

Fig. 5 Cigarette smoke extract inhibits NE and increases mucus accumulation. a Quantification of COPD mucin degradation after incubation at $37^{\circ} \mathrm{C}$ with increasing concentrations of CSC (5-40 mg/mL) for 0,24 and $36 \mathrm{~h}$. b Substrate specific activity of NE $0.33 \mu \mathrm{g} / \mathrm{mL}$ with and without CSC $(5-40 \mathrm{mg} / \mathrm{mL})$. Mucin concentration of the native control was set to $100 \%$

\section{Discussion}

We had anticipated that with an acute exacerbation of COPD, there would be increased inflammation and increased proteases with subsequent degradation of mucins, Thus we were surprised to discover that at the start of a COPD exacerbation there was consistently decreased proteases, increased anti-proteases, and increased mucin stability. A possible explanation for this is that although we evaluated subjects and collected sputa at the start of symptoms of an exacerbation, it is likely that the inciting infection and inflammation had been present for several days. These data might reflect the natural host immune response to decrease the initially observed increased protease activity. Although this is entirely speculative, it would explain these paradoxical results.

One consequence of inhibiting mucin degradation might be increased mucus obstruction, which is considered a hallmark of a COPD exacerbation. It has been reported that persons with COPD have increased mucus synthesis and secretion, and decreased mucus clearance [19, 20,22]. We have reported that in CF sputum, serine proteases degrade mucins after secretion [9]. Bacterial or host inflammatory cell proteases in CF sputum may further contribute to mucin degradation [23]. Delayed mucin degradation in COPD could well be caused by this protease-anti protease imbalance. We report a 5 -fold increase in MUC5AC and a 2 fold increase in MUC5B at the onset of symptoms and even 5-6 weeks later, MUC5AC was 3 times greater in COPD sputa compared to mucus from healthy controls (Fig. 1a, b).

COPD is an inflammatory disease of small airways with increased neutrophil infiltration and NE [14, 30]. Studies performed by other groups suggested that in mucoid COPD sputum no NE was found (NE nM 0.0) $[8,27]$. These observations are similar to our findings, where little or no NE activity was observed in mucoid sputa of COPD subjects at the onset of an exacerbation (Fig. 2a, c). In CF, sputum NE is predominantly bound by DNA and this inhibits proteolytic activity [17]. Much of the DNA in CF sputum originates from neutrophil extracellular traps (NETs). It is speculated that NE-NET formations are reservoirs of active proteases for a possible later release [6]. In CF and COPD sputa the DNA concentration is higher than in normal airway secretions, therefore this NE 
that is bound to DNA is not available for mucin degradation $[9,10,18]$. A1-PI is increased during infection and inflammation and its primary role is to inhibit NE. Consistent with our results (Fig. 2b), during an acute exacerbation of COPD, A1-PI is elevated in sputum [29], serum [7] and in exhaled breath condensate [15]. We did not detect increased NE concentrations or protease activity during an acute COPD exacerbation, which is in agreement with previous studies $[8,27,32]$.

We also show that CSC can inhibit mucin degradation in a dose dependent manner by inhibiting the activity of $\mathrm{NE}$ (Fig. 5b). Thus tobacco smoke and increased DNA might contribute to mucus retention in COPD.

\section{Competing interests}

The authors declare that they have no conflicts of interest with the argument treated in the paper. This work was performed in the Department of Pulmonary Medicine and Critical Care, Philipps-University Marburg, Marburg - Germany.

\section{Authors' contributions}

SC and MOH designed the study; JP, SL, CM, PM and GS all contributed to the clinical and laboratory work for the study; $\mathrm{MOH}$ take responsibility for the integrity of the data in the study and the accuracy of the data analysis; $\mathrm{SC}$ and $\mathrm{MOH}$ analyzed the data and wrote paper; BKR, CV, PM AG and LN contributed to critical review and final approval of the manuscript. All authors read and approved the final manuscript.

\section{Acknowledgements}

This study was supported by the Universities of Giessen and Marburg Lung Center (UGMLC) within the LOEWE program of the state of Hessen and by the German Center for Lung Research (DZL), Germany and by the Denny Hamlin Foundation (BKR).

\section{Author details}

'Department of Pediatrics, Justus Liebig University Giessen, Feulgenstrasse 12, Giessen 35392, Germany. ${ }^{2}$ Department of Internal Medicine, Justus-Liebig-University Giessen, Klinikstrasse-36, Giessen 35392, Germany. ${ }^{3}$ Department of Pulmonary Medicine, Philipps-University Marburg, Baldingerstrasse 1, Marburg 35043, Germany. ${ }^{4}$ Department of Pediatrics, Virginia Commonwealth University School of Medicine, 1001 East Marshall Street, Richmond 23298, VA, USA. ${ }^{5}$ Asklepios Fachkliniken München-Gauting, Robert-Koch-Allee 2, Gauting 82131, Germany. ${ }^{6}$ Member of the Comprehensive Pneumology Center (CPC), Helmholtz Zentrum, Munich, Germany. ${ }^{7}$ Member of the German Centre for Lung Research (DZL), Giessen, Germany. ${ }^{8}$ Member of the European IPF Registry/Biobank, Giessen, Germany. ${ }^{9}$ Lung Clinic Waldhof-Elgershausen, Greifenstein, Germany.

Received: 14 April 2015 Accepted: 1 July 2015

Published online: 15 July 2015

\section{References}

1. Abboud RT, Vimalanathan S. Pathogenesis of COPD. Part I. The role of protease-antiprotease imbalance in emphysema. Int J Tuberc Lung Dis. 2008;12:361-7.

2. Anthonisen NR, Manfreda J, Warren CP, Hershfield ES, Harding GK, Nelson NA. Antibiotic therapy in exacerbations of chronic obstructive pulmonary disease. Ann Intern Med. 1987;106:196-204.

3. Carp H, Miller F, Hoidal JR, Janoff A. Potential mechanism of emphysema: alpha 1-proteinase inhibitor recovered from lungs of cigarette smokers contains oxidized methionine and has decreased elastase inhibitory capacity. Proc Natl Acad Sci U S A. 1982;79:2041-5.

4. Crooks SW, Bayley DL, Hill SL, Stockley RA. Bronchial inflammation in acute bacterial exacerbations of chronic bronchitis: the role of leukotriene B4. Eur Respir J. 2000;15:274-80.

5. Doring G. The role of neutrophil elastase in chronic inflammation. Am J Respir Crit Care Med. 1994;150:\$114-7.
6. Dubois AV, Gauthier A, Brea D, Varaigne F, Diot P, Gauthier F, et al. Influence of DNA on the activities and inhibition of neutrophil serine proteases in cystic fibrosis sputum. Am J Respir Cell Mol Biol. 2012;47:80-6.

7. Ganrot PO, Laurell CB, Eriksson S. Obstructive lung disease and trypsin inhibitors in alpha-1-antitrypsin deficiency. Scand J Clin Lab Invest. 1967;19:205-8.

8. Gompertz S, O'Brien C, Bayley DL, Hill SL, Stockley RA. Changes in bronchial inflammation during acute exacerbations of chronic bronchitis. Eur Respir J. 2001;17:1112-9.

9. Henke MO, John G, Rheineck C, Chillappagari S, Naehrlich L, Rubin BK. Serine proteases degrade airway mucins in cystic fibrosis. Infect Immun. 2011;79:3438-44.

10. Henke MO, Renner A, Huber RM, Seeds MC, Rubin BK. MUC5AC and MUC5B mucins Are decreased in cystic fibrosis airway secretions. Am J Respir Cell Mol Biol. 2004;31:86-91.

11. Hogg JC. Pathophysiology of airflow limitation in chronic obstructive pulmonary disease. Lancet. 2004;364:709-21.

12. Hogg JC, Chu FS, Tan WC, Sin DD, Patel SA, Pare PD, et al. Survival after lung volume reduction in chronic obstructive pulmonary disease: insights from small airway pathology. Am J Respir Crit Care Med. 2007;176:454-9.

13. Hogg JC, Chu F, Utokaparch S, Woods R, Elliott WM, Buzatu L, et al. The nature of small-airway obstruction in chronic obstructive pulmonary disease. N Engl J Med. 2004;350:2645-53.

14. Keatings VM, Barnes PJ. Granulocyte activation markers in induced sputum: comparison between chronic obstructive pulmonary disease, asthma, and normal subjects. Am J Respir Crit Care Med. 1997;155:449-53.

15. Koczulla AR, Noeske S, Herr C, Koepke J, Jorres RA, Nell C, et al. Alpha-1 antitrypsin is elevated in exhaled breath condensate and serum in exacerbated COPD patients. Respir Med. 2012;106:120-6.

16. Moraes TJ, Zurawska JH, Downey GP. Neutrophil granule contents in the pathogenesis of lung injury. Curr Opin Hematol. 2006;13:21-7.

17. Papayannopoulos V, Staab D, Zychlinsky A. Neutrophil elastase enhances sputum solubilization in cystic fibrosis patients receiving DNase therapy. PLoS One. 2011;6:e28526.

18. Picot R, Das I, Reid L. Pus, deoxyribonucleic acid, and sputum viscosity. Thorax. 1978;33:235-42.

19. Prescott $E$, Lange $P$, Vestbo J. Chronic mucus hypersecretion in COPD and death from pulmonary infection. Eur Respir J. 1995;8:1333-8.

20. Ramos FL, Krahnke JS, Kim V. Clinical issues of mucus accumulation in COPD. Int J Chron Obstruct Pulmon Dis. 2014;9:139-50.

21. Reid L. The pathology of emphysema. Postgrad Med. 1966;39:367-73.

22. Rogers DF. Physiology of airway mucus secretion and pathophysiology of hypersecretion. Respir Care. 2007;52:1134-46. discussion 1146-1139.

23. Rose MC, Brown CF, Jacoby 3rd JZ, Lynn WS, Kaufman B. Biochemical properties of tracheobronchial mucins from cystic fibrosis and non-cystic fibrosis individuals. Pediatr Res. 1987;22:545-51.

24. Rose MC, Voynow JA. Respiratory tract mucin genes and mucin glycoproteins in health and disease. Physiol Rev. 2006;86:245-78.

25. Rubin BK, Finegan B, Ramirez O, King M. General anesthesia does not alter the viscoelastic or transport properties of human respiratory mucus. Chest. 1990;98:101-4.

26. Rubin BK, Ramirez O, Zayas JG, Finegan B, King M. Collection and analysis of respiratory mucus from subjects without lung disease. Am Rev Respir Dis. 1990;141:1040-3

27. Sethi S, Wrona C, Eschberger K, Lobbins P, Cai X, Murphy TF. Inflammatory profile of new bacterial strain exacerbations of chronic obstructive pulmonary disease. Am J Respir Crit Care Med. 2008;177:491-7.

28. Stockley RA. Neutrophils and protease/antiprotease imbalance. Am J Respir Crit Care Med. 1999;160:S49-52.

29. Stockley RA, Burnett D. Alpha,-antitrypsin and leukocyte elastase in infected and noninfected sputum. Am Rev Respir Dis. 1979;120:1081-6.

30. Vestbo J, Hurd SS, Agusti AG, Jones PW, Vogelmeier C, Anzueto A, et al. Global strategy for the diagnosis, management, and prevention of chronic obstructive pulmonary disease: GOLD executive summary. Am J Respir Crit Care Med. 2013;187:347-65

31. Vestbo J, Hurd SS, Agusti AG, Jones PW, Vogelmeier C, Anzueto A, et al. Global strategy for the diagnosis, management and prevention of chronic obstructive pulmonary disease, GOLD executive summary. Am J Respir Crit Care Med. 2013;187:347-65

32. White AJ, Gompertz S, Bayley DL, Hill SL, O'Brien C, Unsal I, et al. Resolution of bronchial inflammation is related to bacterial eradication following treatment of exacerbations of chronic bronchitis. Thorax. 2003;58:680-5. 